\title{
Thrombektomie beim Schlaganfall: Schnellste Transporte und Netzwerkbildung erforderlich
}

Stellungnahme der Deutschen Gesellschaft für Neuroradiologie e. V., des Berufsverbands der Deutschen Neuroradiologen e. V., der Deutschen Gesellschaft interdisziplinäre Notfall- und Akutmedizin (DGINA) e. V., der Deutschen Gesellschaft für Neurologie e. V., der Deutschen Schlaganfall-Gesellschaft e. V., der Deutschen Gesellschaft für Interventionelle Radiologie und minimal-invasive Therapie und der Deutschen Röntgengesellschaft e.V.

$\mathrm{Zu}$ Beginn des Jahres wurden auf der International Stroke Conference in Nashville die Ergebnisse dreier Multicenterstudien vorgestellt (SWIFT-PRIME, ESCAPE, EXTENDA-IA), die der minimalinvasiven, mechanischen Thrombektomie mittels
Kathetertechnik (Stent-Retriever-Systeme) eine hohe Wirksamkeit bescheinigten. Für die Therapie des Schlaganfalls bricht mit dieser Studienlage ein neues Zeitalter an. Die endovaskuläre Methode, die bis dato als experimentell eingestuft war, wird nun die führende Rolle in der Akutversorgung ischämischer Schlaganfallpatienten spielen.

Vor diesem Hintergrund spielt die medizinisch richtige Priorisierung von Schlaganfallpatienten im Rettungsdienst eine zentrale Rolle. Die unterzeichnenden Fachgesellschaften und Berufsverbände erklären auf Grundlage oben genannter Studienergebnisse und im Sinne einer qualitativ bestmöglichen Patientenversorgung:

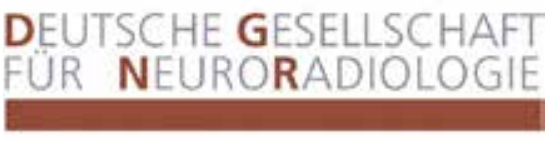

1. Der Primärtransport eines Schlaganfallpatienten im Lyse-Zeitfenster ist grundsätzlich ein Notfall, der mit höchster Priorität unter Nutzung der Sonderrechte unverzüglich zu erfolgen hat. Der Symptombeginn ist stets auf dem Rettungsdienstprotokoll zu vermerken.

2. Bei Patienten mit Indikation zur mechanischen Thrombektomie soll der Transport von der Stroke Unit in eine neuroradiologische Einrichtung mit Thrombektomiebereitschaft als Notfalltransport erfolgen. Dafür ist im Zweifelsfall auch schnellstmöglich ein Hubschrauber zur Verfügung zu stellen, wenn dies den schnellsten Transportweg sicherstellt.

Deutschland bietet aufgrund langjähriger Expertise in der mechanischen Thrombektomie in vielen Zentren und der relativ 
guten Flächenabdeckung mit neuro- und interventionell-radiologischen Einrichtungen im internationalen Vergleich eine gute minimalinvasive Schlaganfallversorgung. Entscheidend für die Sicherung und den Ausbau dieser Qualität sind die Teilnahme an Schlaganfallnetzwerken mit telemedizinischer Infrastruktur sowie der medizinisch korrekte rettungsdienstliche Transport der Patienten oder gegebenfalls des intervenierenden Neuroradiologen.

Die Fachgesellschaften fordern die Politik und verantwortlichen Verwaltungsbehörden auf, die Rettungsdienste der Länder in diese Richtung zu informieren und entsprechende Richtlinien zu erlassen. Die Fachgesellschaften stehen dabei mit fachlichem Rat gerne zur Seite.

Für die Deutsche Gesellschaft für Neuroradiologie e. V. (DGNR):

Prof. Dr. Christoph Groden (Mannheim), Präsident

Für den Berufsverband Deutscher Neuroradiologen e. V. (BDNR):

Prof. Dr. Ansgar Berlis (Augsburg), Präsi-

dent

Für die Deutsche Gesellschaft interventionelle Notfall- und Akutmedizin e.V. (DGI$N A)$ :

Prof. Dr. Christoph Dodt (München), Präsident

Für die Deutsche Gesellschaft für Neurologie e.V. (DGN):

Prof. Dr. Ralf Gold (Bochum), Präsident

Für die Deutsche Schlaganfall-Gesellschaft e.V. (DSG):

Prof. Dr. Gerhard F. Hamann (Günzburg), 1. Vorsitzender

Für die Deutsche Gesellschaft für Interventionelle Radiologie und minimal-invasive Therapie (DeGIR):

Prof. Dr. Arno Bücker

Für die Deutsche Röntgengesellschaft e.V. (DRG):

Prof. Dr. Dierk Vorwerk 